\title{
Sourcing Maturity Self-Assessment
}

\author{
Bert van Zomeren ${ }^{1}$, Alexander Levinson ${ }^{2}$ \\ ${ }^{1}$ SURF, P.O. Box 2290, 3500 GG Utrecht, the Netherlands, vanzomeren@surf.nl \\ ${ }^{2}$ Amsterdam University of Applied Sciences, Amsteldijk 166, 1079 LH Amsterdam, the Netherlands, \\ a.levinson@hva.nl
}

\section{Keywords}

Sourcing process, sourcing maturity, maturity assessment, cloud computing.

\section{ABSTRACT}

There is a growing awareness among the institutions of Higher Education (HE) in the Netherlands that the use of cloud computing could help solve the problems arising from diminishing budgets. Focusing on core business while transferring (parts of) the institutional IT to the cloud could reduce costs and also increase both flexibility and quality.

Moving to the cloud, however, is not without risk. Success is not guaranteed. If an institution is not "cloud-ready", a cloud adventure could turn into a failure and bring disadvantages. Changing to information services from the cloud is actually a sourcing decision. What institutions really need is a mature sourcing process.

SURF, the collaboration organisation of Dutch HE, is helping the institutions to make the transition to cloud services. In 2011 a "Cloud First" IT strategy was developed, which received strong support. In 2012 SURF developed a template and organized workshops to help CIOs and IT directors to formulate an institutional sourcing strategy. Commissioned by SURF, Amsterdam University published a study on the relation between the Patriot Act and Dutch and European Privacy Laws. In collaboration with $\mathrm{CIOs}$ and architects from the institutions a common I-strategy for Dutch HE is under development. This project will also produce common architecture principles and a generic reference architecture. And finally there is a growing interest in "cloud readiness".

\subsection{Sourcing Maturity}

Existing models for general IT maturity (like CMMI) are complex. It is not uncommon for institutions to commission a general IT maturity audit by external auditors, which usually is a time consuming and costly affair. The eSCM-CL model, originated at Carnegie Mellon University, focuses on esourcing capabilities. It discusses 95 best practices, which can be related to different phases of the sourcing process, and to different maturity levels. So far the complexity of this model has discouraged most Dutch institutions from using it in an assessment of sourcing maturity.

Maturity, however, is a success factor for sourcing. Therefore, SURF decided to develop a lightweight sourcing maturity self-assessment, to help institutions start an internal dialogue and set first steps towards increased sourcing maturity.

\subsection{A simple model}

The SURF Sourcing Maturity Model (SSMM) contains 26 items divided in 5 categories. Some of these items are best practices meant to improve the level of execution of the sourcing process. Other items can be seen as conditions or guidelines for developing a "cloud-ready" organization.

The five categories are related to consecutive phases of the sourcing process, i.e. 1) Preparations (and conditions), 2) Demand articulation, 3) Market exploration and decision making, 4) Provider selection and implementation and 5) Exploration and evaluation.

Each item consists of a subject and a short description of an ideal situation (maturity level 5) regarding this subject. The respondent is asked to assign a score to each item in conformance with the usual maturity levels, i.e. 1) initial, 2) managed, 3) defined, 4) quantitatively managed, and 5) optimizing. Some examples of the items are 
$>$ Strict separation of demand and supply;

Availability of an institutional sourcing strategy;

$>$ The quality of IT-governance;

$>$ The ability to "manage" vendors;

$>$ The quality of the institutional architecture process;

$>$ The ability to manage projects.

\subsection{Assessment process}

Because the main purpose of the self-assessment is to create collective awareness of sourcing maturity issues (and their solutions) within the organization, the institutions are advised to execute the assessment as follows.

$>$ Key-players in the sourcing process are identified;

$>$ Each key-player individually performs the self-assessment;

> Individual results are analyzed; large differences and similarities are pinpointed;

The key-players meet, discuss the results and decide on actions to improve maturity.

This will increase the efficiency and the chances of success of the sourcing activities.

\subsection{Tool and validation}

The first version of the assessment tool is available in the form of a spreadsheet in Dutch. The tool can be used to register individual results, to summarize individual results, and to combine individual results into a graphical impression of the institution's sourcing maturity. A very important diagram shows mean and range of the scores per category, averaged over the individual respondents.

The first results are positive, obtained in pilots at two Dutch institutions.

\subsection{Further work}

More pilots will be conducted, several institutions have shown interest. The second version will be implemented, both as a spreadsheet as well as a web application, which will enable central storage of institutional results, and benchmarking against other institutions. An English version of the tool will be made available to the international community.

\subsection{Discussion}

First results indicate that institutional staff understand the questions and are able to score the items on the usual maturity scale of 1 to 5 . Seen in this perspective, the self-assessment can be called a success. However, determining a sourcing maturity score in itself does not give any guarantees nor does it produce any extra value. The assessment serves as a diagnostic tool by indicating strong and weak points with respect to the sourcing process. Added value will only be produced if the meeting of key staff, after discussing the assessment results, is able to decide on improvement actions.

\section{ABOUT THE AUTHORS}

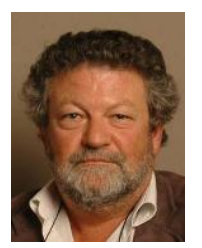

Bert van Zomeren has a degree in mathematical statistics. He worked first as a scientific programmer at Leiden University and then for more than 25 years at Delft University as an assistant professor, information manager and enterprise architect consecutively. He is a member of the SURF Taskforce Cloud since September 2011.

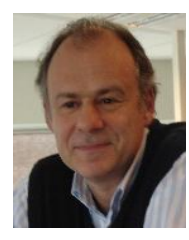

Alexander Levinson is, since 2008, a Central Service and Sourcing manager at the University of Applied Sciences of Amsterdam. He participated in creating and analyzing general sourcing strategies for institutions in Dutch HE. Prior to joining the University he worked as a freelance consultant and advisor in the area of IT Management and Procurement for various international companies in the financial sector, government, telecom and transport, especially in Belgium, Russia, France and the UK. 